\title{
A MINIMUM HOP ROUTING ALGORITHM FOR MULTIHOP CELLULAR NETWORKS
}

\author{
Mark DeFaria and Elvino S. Sousa
}

\begin{abstract}
This paper presents a minimum hop routing algorithm for the multihop cellular CDMA network. A mechanism to update routing paths in case of network topology change is also developed. Mobile terminals will be separated into two groups and each group will only transmit on one of two timeslots so that terminals do not have to transmit and receive simultaneously on the same frequency band. The routing algorithm in this paper is designed in order to optimize criteria including SNIR, delay, power consumption, and network lifetime. Simulation is used to evaluate these criteria for the multihop cellular network and its performance is compared with the traditional cellular network. Simulations show that the total power consumption in the multihop cellular network is much lower than a traditional cellular network with a capacity that is nearly as high. Simulations also show that the network lifetime of the multihop cellular network is much higher than that of a traditional cellular network.
\end{abstract}

Keywords: Multihop Cellular Networks, Routing Algorithms, Ad hoc networks.

Resumo - Este artigo apresenta um protocolo de roteamento para redes multihop celulares CDMA com nmero mnimo de saltos. apresentado tambm um mecanismo para atualizar os caminhos de roteamento quando a topologia da rede alterada. Terminais mveis sero separados em dois grupos, e cada grupo transmitir apenas em um a cada dois time-slots, tal que os terminais no tero que transmitir e receber simultaneamente na mesma faixa de freqncia. $\mathrm{O}$ algoritmo de roteamento neste artigo projetado para otimizar critrios incluindo SNIR, atraso, consumo de potncia e tempo de vida da rede. Simulao usada para determinar estes critrios para uma rede multihop celular, e seu desempenho comparado com o de uma rede celular tradicional. Os resultados mostram que a potncia total consumida em uma rede multihop celular muito menor que em uma rede celular tradicional, ambas com capacidades prximas. Os resultados tambm mostram que o tempo de vida de uma rede multihop celular muito maior do que aquele de uma rede celular tradicional.

Palavras-chave: Redes celulares multihop, algoritmos de roteamento, redes ad hoc.

\section{INTRODUCTION}

Mark DeFaria and Elvino S. Sousa are with the Department of Electrical and Computer Engineering, University of Toronto, Toronto, ON M5S 3G4, Canada. E-mails: defaria@comm.utoronto.ca, sousa@comm.utoronto.ca



Figure 1. Cellular Networks, Ad hoc Networks and Multihop Cellular Networks

In recent years, the continuing demand for mobile communication systems with higher capacity, lower energy consumption, and larger coverage has motivated research in the area of multihop cellular networks. A multihop cellular network exploits advantages of both traditional cellular networks and ad hoc networks by employing a hybrid architecture. In a multihop cellular network, the maximum transmission power of mobile terminals as well as base stations will be lower than in a traditional cellular network and therefore they will no longer necessarily be within transmission range of one another. Therefore, other mobile terminals will be used to forward packets to and from the base station using multiple hops, as in an ad hoc network. A single-hop architecture will be de_ned as a system inwhich transmissions from any mobile terminal can reach a base station in a single hop. A multihop architecture will be de_ned as an architecture in which not every mobile terminal is within range of a base station and where other terminals may be required to forward transmissions to a base station. Traditional cellular networks employ a single hop architecture whereas multihop cellular networks employ a multiple hop architecture. Figure 1 illustrates the differences between traditional cellular networks, mobile ad hoc networks and multihop cellular networks. Mobile terminals in a multihop cellular networks require a smaller amount of transmission power than traditional cellular networks because the maximum transmission range of each terminal is reduced and multihop outing is used. This 
reduction in energy consumption will lead to an increase in battery life. Moreover, the eduction in transmit power has the effect of decreasing the overall interference. The majority of the intercell interference at a base station is the result of the transmissions from mobile terminals near the boundaries of neighboring cells. However, since the transmission power of terminals near boundaries of neighboring cells will be reduced in a multihop cellular, the intercell interference may also be decreased. Therefore, multihop cellular networks have the potential to provide higher Signal to Noise and Interference Ratios(SNIR) than traditional cellular networks. Finally, the coverage in a multihop cellular network will be increased because mobile terminals that are too far from any base station will still be able to communicate with it by having its transmissions forwarded to it by other mobile terminals. Furthermore, coverage would also be improved because any mobile terminal that is caught in a deep shadow fade with respect to the base station would still be able to communicate by relaying its data to one of its neighbors. Finding the paths that a packet must follow to get from its source to its destination is one of the most difficult tasks when designing a network. Routing paths from each mobile terminal to a base station need to be found in a multihop cellular network because each mobile terminal is not necessarily within transmission range of the base station. Therefore an approach to routing more similar to that in a traditional ad hoc network needs to be investigated. Although many different routing strategies have been designed and compared for traditional ad hoc networks, the same cannot be said for multihop cellular networks. This is what makes routing one of the main dif_culties when designing a multihop cellular network. In a multihop cellular network, it is very important to design a distributed algorithm that nds routing paths from each mobile terminal to the base station as to minimize total energy consumption, minimize interference and maximize the overall lifetime of the network. These goals motivate the use of a minimum hop routing algorithm for several reasons. The total energy consumption when using a minimum hop routing algorithm will be small because a path with the smallest number of hops will be chosen. Interference will be minimized because a minimum hop routing algorithm will ensure that interfering terminals are as far away from one another as possible. The lifetime of the network will potentially be large because the overall energy consumption is low. Moreover, the end-to-end delay will be small because paths with a minimum number of hops will be chosen. Several centralized routing algorithms have been proposed for multihop cellular networks in previous works [?][?]. However, in a real network it is not realistic to assume that any terminal has an entire view of the network, and therefore a distributed routing algorithm is required. In this paper, we propose a fast, distributed routing algorithm with the goal of a high overall SNIR, while maintaining a low overall energy consumption and a large network lifetime. Simulation and analysis have shown that the overall power consumption can be decreased by up to $18 \mathrm{~dB}$ as compared to a traditional cellular network. The structure of this paper is as follows. In section 2, we will present related research that has been performed in the area of multihop cellular networks. This paper continues in section 3 with a description of the system model. In section 4 , we introduce the minimum hop routing algorithm for the multihop cellular network. In section 5 , we present the results of our simulations and analysis. Finally, the paper is concluded and an outline of future work is given in section 6 . The multihop cellular network was investigated by $\mathrm{Lin}$ and $\mathrm{Hsu}$ [?] in 2000. In this paper they compared the throughput of a multihop cellular network with that of a single hop cellular network. This paper assumed a Request To Send/Clear To Send(RTS/CTS) multiple access method and did not require the base station to forward packets for intracellular calls. Traf_c locality was de_ned as the percentage of packets that were destined to other mobile terminals within the cell. Their simulations showed that the overall capacity of the multihop cellular network was higher than that of a traditional single hop cellular network when traf_c locality is high. This is is because the bottleneck that would normally occur near the base station was decreased because it was no longer in charge of forwarding intracellular calls. However, this paper used the all-pairs shortest path algorithm, Floyd-Warshall algorithm [?] to pre-compute the routing tables for each mobile terminal. In a distributed system, this would not be possible and therefore a more realistic routing algorithm is required. Further research was performed on the multihop cellular network using CDMA as the multiple access method and the base station to forward every packet[?]. Pepe presented a centralized SIR/Power Routing Algorithm similar to Dijkstra's algorithm in an attempt to minimize an overall network cost function. Research was also performed by Kusuma in which he presented a centralized algorithm that found the minimum power routing paths for all nodes in the network[?]. However, it is not realistic to assume that an entire view of the network is known, and therefore a distributed routing algorithm is required. Furthermore, the effects of intercell interference were not taken into account in their results. In this paper, we will also be using CDMA as our multiple access method and the base station will be used to forward every packet. Allowing the base station to be used to forward every packet simpli_es the routing algorithm because routes only need to be found from each terminal to its base station rather than to every other terminal within the cell. A duplex system is a system where users can transmit and receive simultaneously. Duplexing is necessary in a traditional cellular network because two way communication is involved and it is essential for the delay to be small for voice. Frequency division duplexing(FDD) involves the division of the frequency spectrum into two distinct bands to obtain a full duplex system. FDD is used in a traditional cellular system with mobile terminals transmitting packets to the base station on the low frequency band and receiving packets from the base station on the high band. Base stations, on the other hand transmit on the high band and receive on the low band. This cannot be done in a multihop cellular network because not only do mobile terminals have to receive packets from a base station but they also have to receive packets from other mobile terminals. Therefore, mobile terminals will have to listen for incoming transmissions on several channels and also make transmissions on several channels. A mechanism where terminals would be separated into two distinct groups was developed in [?]. This mechanism allowed that 
during half of the time, one group of terminals would be transmitting while the other group of terminals were receiving. The other half of the time, the group that was transmitting will now be receiving and the group of terminals that was receiving will now be transmitting. A scheme similar to this will be used in this paper to allow us to tackle the problem that mobile terminals will have to transmit as well as receive on multiple frequency bands.

\section{MULTIHOP CELLULAR NETWORK MODEL}

The multihop cellular network will be analyzed using the following model. The total number of mobile terminals in a cell will be denoted by $M$. The radius of the cell will be denoted by $\mathrm{R}$. Each mobile terminal will be referred to by a value from $\mathrm{i}=1 ;:: ; \mathrm{M}$. The base station will be referred to by $a$ value $i=0$. Mobile terminal $i$ will forward its transmissions towards the base station using a power $\mathrm{Pi}$. The path loss experienced by a transmission from a source terminal $I$ and destination terminal $j$ will be modelled using Gij. The effects of shadowing from source terminal $i$ to destination terminal $\mathrm{j}$ on a transmission will be modelled using $\mathrm{ij}$. The values of $\mathrm{ij}$ will be correlated for terminals that are close to one another. The received power for a destination mobile terminal $j$ from a source terminal $i$ is equal to $\mathrm{Pi}$ _ Gij _ $\mathrm{ij}$. A destination mobile terminal $\mathrm{j}$ will be said to be within transmission range of a source mobile terminal $\mathbf{i}$ when the received power $\mathrm{Pi}_{\text {_ }} \mathrm{Gij}$ _ $\mathrm{ij}_{\mathrm{j}}$ is above a certain threshold_required to decode the transmission with low probability of error. Figure 2 illustrates how transmissions are made from mobile terminals to the base station along with the power at each receiver.



\section{Figure 2. Received Power at a Mobile Terminal}

In a multihop cellular network, the transmission range of each mobile terminal is decreased as compared to a traditional cellular network. The maximum transmission range for the multihop cellular network will be reduced to $1 \mathrm{~K}$ of the maximum transmission range in a traditional single hop cellular network. Packets from mobile terminals that are further away from the base station will need to be forwarded by other mobile terminals. Each transmission will be referred to as a hop. Terminals that are h hops away from the base station will be said to be part of layer h. Frequency division duplexing will be used as in CDMA2000 to separate transmissions in the forward and the reverse link. One reason for this is that it will allow for easier integration to the current infrastructure which currently uses a two frequency FDD system. FDD is also a good technique for the network if it is to be used for twoway communication that requires small delay such as voice. Base stations will be designed to receive only on the low band as in a traditional cellular system. Mobile terminals will have to transmit not only to the base station but to other mobile terminals. Therefore, mobile terminals will be designed so that they can transmit and receive on both the high frequency band and the low frequency band. Two timeslots will be used so that mobile terminals do not have to transmit and receive simultaneously on the same frequency band. This mechanism will be discussed further in Section 3.3. It will be assumed that mobile terminals within a couple of hops of one another are synchronized so that transmissions within two distinct timeslots is possible. CDMA will be used as the multiple access technique with a processing gain denoted by $\mathrm{N}$. Also station mobility is neglected to reduce the complexity of the simulations as the simulation results in [?] show that mobility of terminals may have a small effect on overall system throughput. Power control is used so that the received SNIR of signals destined to a given receiver is equal. Each mobile terminal will generate the same amount of traf_c. Each mobile terminal will generate Poisson distributed traf_c with a rate of _ packets per timeslot. Mobile terminals will be able to simultaneously receive from multiple terminals. They will also be able to simultaneously transmit to multiple terminals using different spreading codes. Mobile terminals will have the ability to adjust the transmit power to each receiver independently. Mobile terminals will also have the ability to simultaneously transmit multiple packets to each receiver. Mobile terminals will not be able to transmit and receive simultaneously on the same frequency band. This is because the transmission power of a terminal will be much higher than the received power and too much interference would be created. The interference created by other transmissions will be treated as independent additive noise [?].

\subsection{SPREADING CODES}

In a multihop cellular network, the base station will be in charge of distributing spreading codes to mobile terminals. The distribution of spreading codes will happen during the discovery of routing paths and this will be further discussed in Section 4. As a perfectly synchronized environment is impossible to create, a spreading code scheme comprised of non-orthogonal spreading code sequences will be used. This will be true both for transmissions from a mobile terminal towards the base station and in the reverse direction. In a traditional CDMA system, non-orthogonal codes are used only on the reverse link. The reason that orthogonal codes can be used on the forward link in a traditional CDMA system but not in a multihop cellular network is that in a traditional cellular network, all transmissions on the forward link emanate only from the base station. This differs in a multihop cellular network because in this system, every mobile terminal will also have to transmit on the forward link. Therefore non-orthogonal spreading sequences will be used for transmissions on the reverse link as well as for transmissions on the forward link. In an asynchronous CDMA system there are usually enough spreading code sequences to be assigned to all mobile terminals within a cell. Each mobile terminal will therefore be assigned a unique spreading code sequence by 
the base station. In fact, terminals that have to forward packets for other terminals will be assigned several spreading codes. Each mobile terminal will then be able to make transmissions using a unique non-orthogonal spreading code sequence determined by the base station in a manner similar to the reverse link of a traditional cellular system.

\subsection{TRANSMISSIONS IN TIMESLOTS}

In a multihop cellular network, a mechanism needs to be developed to handle the problem that mobile terminals will have to make transmissions as well as listen for transmissions on multiple frequency bands. Therefore, the physical layer of mobile terminals in a multihop cellular network will have to be modi_ed from that in the traditional cellular network. Also due to the fact that there is a high power difference between signals being transmitted and signals being received at a terminal, it is impractical for a terminal to have transmissions and receptions on the same frequency band at the same time. Therefore a mechanism needs to be developed that handles this problem. This mechanism will work by separating terminals into two groups[?]. Two timeslots will be used. During one timeslot, mobile terminals within one group will be making transmissions while the terminals within the other group are listening and waiting for transmissions. During the other timeslot, the mobile terminals that were making transmissions will now be listening and the mobile terminals that were listening will now be creating transmissions. It is necessary that mobile terminals within a few hops of each other are synchronized so that minimal packet collision will occur. The manner in which terminals are to be grouped still must be discussed. Terminals will be grouped up in a manner so that no terminal will be sending any information to a destination mobile terminal on the same frequency band that the destination is using to make a transmission. The use of the layer number of each mobile terminal to group mobile terminals will be the perfect mechanism to accomplish this where the layer number was de_ned as the number of hops needed to reach the base station. Therefore, the mechanism will be that any mobile terminal in an even numbered layer should be transmitting in the _rst timeslot, whereas any mobile terminal within an odd numbered layer should be receiving in the _rst timeslot. In the second timeslot, all mobile terminals in an odd numbered layer should be transmitting whereas all mobile terminals in an even numbered layer should be receiving. In subsequent timeslots, the groups of receiving mobile terminals and the groups of transmitting terminals will alternate. This mechanism will ensure that terminals will never be transmitting and receiving simultaneously on the same frequency band. It is possible that traf_c from the base station to the mobile terminals is higher than the traf_c from the mobile terminals to the base station. Because mobile terminals will now be able to transmit and receive on both frequency bands, this architecture allows for the possibility to allow higher traf_crates on the forward link(or the reverse link). If asymmetric traf_c existed within the network, at certain times, both frequency bands could be used to transmit packets on the forward link simultaneously. An ef_cient scheduling protocol would need to be designed for this case. However, this paper will only focus on symmetric traf_c.

\section{MINIMUM HOP ROUTING ALGORITHM}

Routing from a mobile terminal to its base station is a difcult task in a multihop cellular network because transmissions from a mobile terminal cannot reach a base station in a single hop. Therefore a distributed approach to routing must be taken as is done in an ad hoc network. This involves _nding routing paths from all mobile terminals to their respective base stations. Once routing paths are found, there must also be a mechanism for the paths to be updated. An update maybe needed when there is a change in the location of a mobile terminal or an increase of the interference at the receiver.

\subsection{ROUTING PATH DETERMINATION}

Initially, when a mobile terminal is activated, it is completely unaware of its location. In a typical CDMA cellular network, a mobile terminal would wait to hear a pilot signal from a base station. The mobile terminal would then know where to forward its outgoing transmissions. However, in a multihop cellular network the base station and the mobile terminals' transmission power will be reduced and therefore they will no longer necessarily be within transmission range. For this reason, a different mechanism will have to be used so that mobile terminals can transmit packets to the base station as well as receive packets from it. In a multihop cellular network, when a mobile terminal wants to make a transmission, it will use other mobile terminals within the cell to forward its transmissions to the base station. The base station will then use mobile terminals to forward the packets to the destination. Therefore a mechanism needs to be developed which determines which mobile terminals will be used in the routing paths to the base station for packets emanating from each mobile terminal and vice versa. The network topology of a multihop cellular network will be de_ned as follows. A graph can be formed with the existence of links between mobile terminals when terminals are within transmission range of one another. Using a subset of these links, routing paths will be found from every mobile terminal to its base station and vice versa.

\subsubsection{MULTIHOP CELLULAR ROUTE DETERMINATION ALGORITHM}

The multihop cellular route determination algorithm will be designed to _nd routes from all mobile terminals to their respective base stations. One important property for a routing algorithm is that the number of hops from source to 
destination in the routes is small. This is important because a small number of hops implies a small end-to-end delay for each packet. In fact, the route determination algorithm proposed in this paper creates routes with a minimum number of hops to the base station, given the position of the mobile terminals and their maximum transmission range. This is because the routing paths are determined starting from the base station and links between terminals are chosen so that the number of hops is minimized. Also, choosing minimum hop routes will minimize the interference at the base station. This is important because it will allow the SNIR to be maximized at the base station which is the bottleneck of the system. The parent of a source mobile terminal will be de_ned as the terminal to whom the source will transmit its packets destined for the base station. The children of a parent mobile terminal will be de_ned as all the terminals that transmit their packets destined for the base station to the parent. This routing algorithm has the property that it will form routing paths that have a tree-like structure, where each mobile terminal can have many children but only one parent and the base station is the root of the tree. To _nd the routing paths for all mobile terminals within a cell to the base station, the route discovery will begin at the base station. The base station will send a message to every mobile terminal within its range to inform the mobile that they are within range of the base station. These mobile terminals will belong to layer 1 because they are within one hop of the base station. These mobile terminals will also be referred to as the children of the base station and the base station will be referred to as the parent of the mobile terminals. The mobile terminals will then reply to the base station to inform it that they are within transmission range of the base station. All mobile terminals could begin to transmit immediately upon receiving the message from the base station. However, to minimize collision between the replies to the base station, an ALOHA type random access scheme will be used so that all mobile terminals transmissions begin at a random time. This ALOHA type random access scheme will be discussed further in Section 4.1.3. The base station will then acknowledge the reply with a message to each mobile terminal within its range indicating the receipt of the mobile terminals' messages. The next hop for transmissions towards the base station for any mobile terminal will be its parent. The next hop towards the base station for each mobile terminal in layer 1 will therefore be the base station. The base station will then send a message to the mobile terminals in layer 1, assigning them a timeslot and a spreading code. This concludes the route determination for all mobile terminals within transmission range of the base station. The routing paths for the remaining mobile terminals, that are not within transmission range of the base station, still need to be computed. To accomplish this, each mobile terminal in layer 1 will send a message at its assigned timeslot to all mobile terminals within its range. Any mobile terminal that is not part of layer 1, that receives a message from a terminal in layer 1 , will become part of layer 2 . After every terminal in layer 1 has _nished its transmission, each mobile terminal in layer 2 will choose one terminal in layer 1 , from which it has received a transmission, to be its parent based on speci_c criteriatto be discussed in the subsequent section). The mobile terminal will reply to the message of the chosen parent once again using an ALOHA type scheme. For example one possible criterion could be largest received SNIR and another could be lowest received SNIR above a certain threshold. The parent of the mobile terminal in layer 2 will be its next hop towards the base station. Any mobile terminal in layer 1 that is chosen to be a parent will acknowledge the receipt of the message to the mobile in layer 2 using its spreading code. Parent terminals will also inform the base station about the existence of their children using their spreading codes. The base station will then ssign spreading codes and timeslots to terminals in layer 2 and will forward this information to the parents of the terminals in layer 2 who will in turn forward the information to the mobile terminals in layer 2. Repeating this algorithm for mobile terminals in higher layers, complete routing tables can be calculated for the entire multihop cellular network. The route determination algorithm is summarized explicitly below:

1. Base station sends a packet to every mobile terminal within its range to initiate the route discovery. Packet Contains: SourceID=baseID, Layer $=1$, Message Type $=1$

2. Mobile terminals that receive a message are within range of the base station and will reply to the base station using ALOHA with transmissions starting at random times. Packet Contains: SourceID $=$ MobileID, DestinationID $=$ BaseID

3. Base station will record the direct link between the terminals and the base station and send a message to acknowledge each mobile terminal that replied to it in step 2. Packet Contains: SourceID=baseID, DestinationIDs = all mobile terminals that replied, MessageType $=2$

4. In the small likelihood that there was a collision in step 2 , mobiles terminals will discover this when they are not acknowledged in step 3 . In this case those terminals will retransmit message in step 2 using ALOHA and go back to step 3. Otherwise continue to step 5.

5. Base station sends a message informing all mobiles in layer 1 of their spreading code and timeslot. Packet Contains: SourceID = BaseID, MessageType $=3$, destinationid $=$ MobileID, Timeslot, SpreadingCode

6. Every mobile terminal in layer 1 will then send a packet to any mobile terminals within its range(who are not in layer 1) at its timeslot informing them that they are in layer 2. Packet Contains: SourceID $=$ MobileID(in layer 1), Layer $=2$, MessageType $=1$, TotalTimeslots, SourceSpreadingCode

7. After all terminals in layer 1 have transmitted, each terminal in layer 2 will choose one of the mobile terminals that transmitted to it(based on criteria to be discussed in the next section) in step 6 and send a reply using an ALOHA type scheme. This mobile terminal will be known as its parent and will be chosen based on its criteria. Packet Contains: SourceID $=$ MobileID(in layer 2), DestinationID(in layer 1)

8. Any mobile terminal in layer 1 that received a reply in step 7 will send an acknowledgement message to its child. Packet Contains: SourceID $=$ MobileID(in layer1), DestinationIDs $=$ all mobiles that replied, MessageType $=2$ 9. In the small likelihood that there was a collision in step 7 , mobile terminals will discover this when they are not 
acknowledged. In this case retransmit the message in step 7 and go back to step 8. Otherwise continue to step 10 .

10. Mobile terminals in layer 1 will relay the information about mobile terminals in layer 2 to the base station using their spreading codes. Packet Contains : SourceID = MobileID(in layer1), DestinationID = BaseID, ReachesID = MobileIDs(in layer2), Layer=2

11. Base station records the existence of the link and assigns spreading codes and timeslots to terminals in layer 2.Base station will transmit this information to terminals in layer 1. Packet Contains : SourceID = BaseID, NextHopID = MobileID(in layer 1), DestinationID = MobileID(in layer 2), SpreadingCode, Timeslot, MessageType $=3$

12. Terminals in layer 1 will forward packet from step 11 to terminals in layer 2 .

13. Process will continue now with mobile terminals in layer 2 discovering terminals in layer 3 by returning to step 6 and following the same process. This process will repeat to discover higher layered terminals until no new mobile terminals are found. The route determination algorithm will have converged when this occurs.

\subsubsection{CRITERIA FOR ROUTE DETERMINATION ALGORITHM}

The routing algorithm described is very _exible because it allows routing paths to be chosen differently depending on how a parameter is chosen. This parameter will allow different terminals to be chosen to forward packets. This choice will depend on the criteria that is important to the system in question. The routing algorithm can function so that routing paths that attempt to minimize the distance between mobile terminals are chosen. This is accomplished by picking the mobile terminal with the highest received SNIR in step 7 of the route determination algorithm. One might choose to pick this criterion because the fact that it has a high SNIR will allow us to conserve battery power by lowering the transmission power of that mobile terminal. This criteria will be referred to as the minimum distance criteria. The routing algorithm can also function so that routing paths are chosen to maximize the distance between mobile terminals while still obtaining a suf_cient SNIR needed to properly decode the signals. This criteria may lead to greater fairness because a greater number of mobile terminals will be in charge of forwarding packets. This could lead to a larger overall network lifetime which will decrease the number of times that routing paths will need to be updated. This criteria will be referred to as the maximum distance criteria. The routing algorithm can also function so that mobile terminals that have a low remaining battery power are not chosen to act as a parent in a routing path. This will lower the number of times that the routing paths need to be updated. Also some mobile terminals may choose not to want to act as routers for other terminals' messages. Another possibility would be to select several routing paths and to transmit on multiple paths. This genre of multipath routing provides path diversity which could lead to higher capacity and a smaller necessity to recalculate routing paths due to network topology changes.
The performance of the multihop cellular network when using the minimum and maximum distance criteria will be fully simulated and compared in Section 5. Another good property of this routing algorithm is that it provides a minimum number of hops from each mobile terminal to the base station given the maximum transmission range of the terminals. This is good because having an algorithm with a minimum number of hops will minimize end-to-end delay and minimize interference between users. However, if load balancing is a concern, the algorithm can easily be modied to allow a mobile terminal to be chosen as a parent only when it has not already reached its maximum number of children. In this case, a load balancing scheme would be enforced and the algorithm would not necessarily provide routes with the minimum number of hops.

\subsubsection{ROUTE DETERMINATION ALGORITHM BASED ON ALOHA}

In the route determination algorithm, a mechanism similar to unslotted ALOHA[?] is used to ensure that packet collision is minimized. When mobile terminals are newly discovered and reply to the messages as in step 2 and step 7 of the route determination algorithm, it is possible that their messages will collide. Therefore instead of terminals ending their messages immediately, they will wait for a random time $\mathrm{Tw}$ before sending their messages. Tmax will be dened as the maximum time that a terminal can wait to begin transmitting its message. The value of $T w$ will be uniformly distributed between 0 and Tmax. We will de_ne $\mathrm{T}$ as a scaled version of time equal to the time it takes to transmit one packet.

$$
T=\frac{L}{R}
$$

$\mathrm{L}$ is the length of a packet and $\mathrm{R}$ is the transmission rate of the link. Assume there are $n$ terminals in a layer. We will de_ne frame size $\mathrm{Lf}$ as being equal to $\mathrm{Tmax} T$. Lf is the total number of packets that could be transmitted if mobile terminals were synchronized. Tmax should be chosen so that $\mathrm{Lf}$ is a value ranging from 10 to 100 times larger than the number of mobile terminals in a layer. Therefore there will be space for more than 10 to 100 times more transmissions than are actually made so that the collision probability is minimized. The probability of no collision in this case is:

$$
P_{n \text { andimin }}=\prod_{k=1}^{n-1}\left(1-\frac{2 k}{L f}\right)
$$

\subsubsection{ROUTE DETERMINATION ALGORITHM EXAMPLE}

In this section we will look at an example of a route discovery inside a multihop cellular network using the route 
etermination algorithm. Initially the network topology of any multihop cellular network will be unknown. The base station will initiate the route determination algorithm and at the conclusion of step 5 , the routes for the mobile terminals within one hop of the base station will be determined. This is shown in Figure 3. Note that the circle around the base station is used to illustrate its maximum transmission range. In reality, the transmission range will not have a perfectly circular shape due to shadowing.

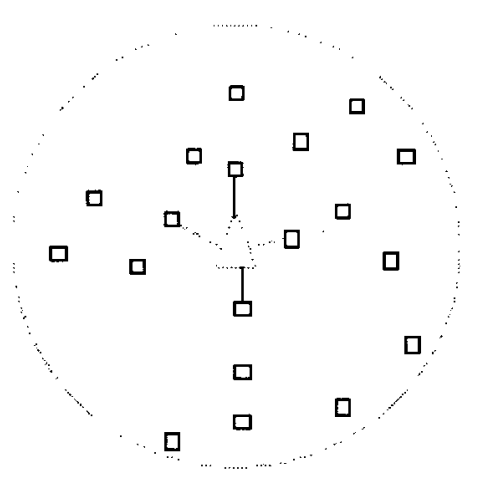

Figure 3. Example of Route Determination Algorithm for Layer 1

Figure 4 illustrates the routes that have been discovered at the conclusion of step 12 of the route determination algorithm. Notice that any mobile terminal that is within range of a terminal in layer 1 becomes part of layer 2 . Also note that the criterion used to select which mobile terminal should become a parent, when two or more terminals are within range, is to choose the one with the maximal received power, illustrated pictorially by choosing the closest mobile terminal.

The route determination algorithm will loop back to step 6 to determine the routes for the mobile terminals within three



Figure 4. Example of Route Determination Algorithm for Layer 2

hops of the base station. This is illustrated in Figure 5.

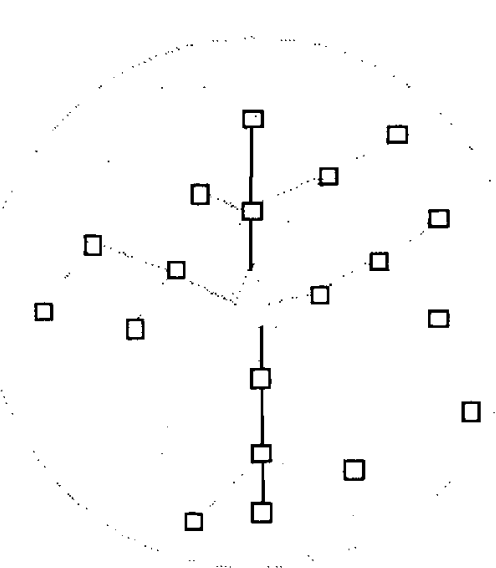

Figure 5. Example of Route Determination Algorithm for Layer 3

The route determination algorithm will loop back to step 6 to determine the routes for the mobile terminals within four hops of the base station. This is illustrated in Figure 6 .

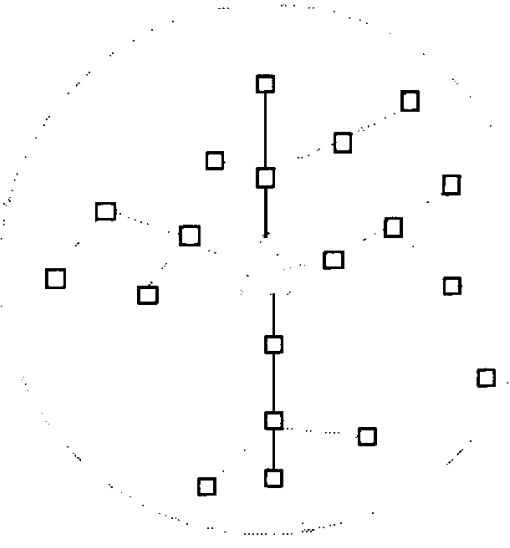

Figure 6. Example of Route Determination Algorithm for Layer 4

The route determination algorithm will loop back one more time to step 6 to attempt to _nd any other mobile terminals. Since all terminals have already found a parent, the algorithm will have converged and the route determination process will be complete. Therefore using this algorithm, routing paths will be found for any mobile terminal that is within range(directly or indirectly) of the base station. Also it can be observed that a tree structure is formed using this algorithm with the base station being the root. The _nal routing paths for all nodes are shown in Figure 7 . Note that the terminals have been separated into two groups using this algorithm. The nodes that have an odd number of hops to reach the base station denoted by a square, and the terminals that have an even number of hops to reach the base station denoted by a circle. Formation of groups will allow mobile terminals to transmit and receive in both the high and low frequency using two different timeslots as was previously discussed. Also note that the layer number is indicated for each terminal. 


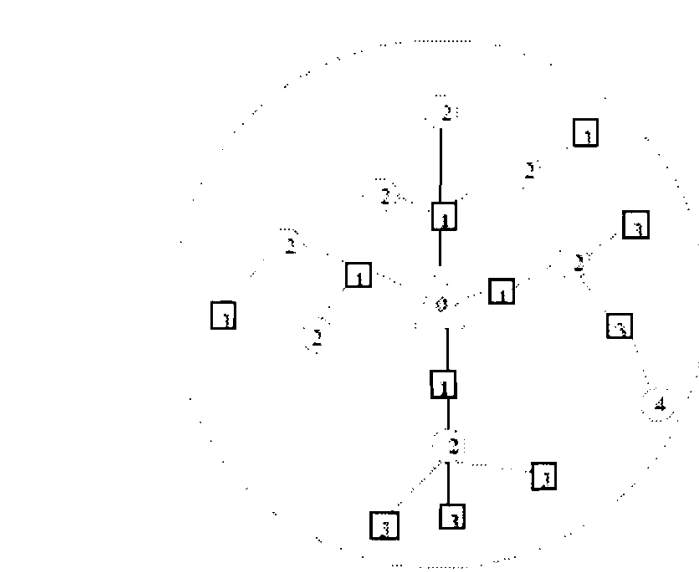

Figure 7. Final Routing Topology using Route Determination Algorithm

\subsection{ROUTING PATH UPDATES}

Interference between terminals is constantly changing and the locations of mobile terminals may also change. When these changes occur, terminals that used to be within transmission range of one another may no longer be. Also new mobile terminals may be activated or existing terminals may deactivate at any time. Therefore a mechanism needs to be designed so that routing paths are updated due to these possible changes in the network topology.

\subsubsection{CHECKING FOR A BROKEN LINK}

Links can break at any time due to the motion of mobile terminals or because of changing amounts of interference between a parent and a child. Therefore every so often, it is imperative that the routing paths are updated. We will de_ne $\mathrm{Tu}$ as the time between subsequent updates of the routing tables in our ultihop cellular network. The value of Tu will be large if there is little or no motion in the mobile terminals in the network. However $\mathrm{Tu}$ would be rather small, when the mobility of the mobile terminals is large. The route update algorithm will work as such. Every Tu seconds, each mobile terminal will send a packet to its parent asking it if they are still within transmission range. If the parent receives the message, it will reply to its hild telling him that a link still exists. Therefore, if the cknowledgement is received the child knows that its link to its parent is still valid. However, if no acknowledgement is received, the mobile terminal will know that the link to its parent is broken and this terminal will be referred to as parentless. In order to _nd a new parent, it will send a packet to every terminal within its range stating that its link has been broken. Any terminal that receives this packet, other than the parentless terminal's children, will reply to the message stating that it can act as the terminal's new parent. The parentless terminal will then choose to acknowledge the terminal with the lowest layer number based on its criterion(who will in turn forward the information to the base station). The base station will then then send a packet to the terminal's old parent to informit that the parentless terminal is no longer its child. The parentless terminal will also transmit a packet to its children to inform them of their updated layer values(who will forward this information to their children and so on). An explicit summary of the route update algorithm is shown below:

1. Every Tu seconds, each mobile terminal will send a packet to its parent to check if the link still exists. Packet Contains : SourceID $=$ ChildID, DestinationID

$=$ ParentID, MessageType $=11$

2. If the parent receives the message in step 1 , it will reply to its child to reassure it that the link exists Packet Contains : SourceID $=$ ParentID, DestinationID $=$ ChildID, MessageType $=12$ Note: If the child receives a packet in step 2, it knows the link is not broken and the algorithm ends here. If the child did not receive a packet in step 2, it knows that there is a broken link and continues to step 3 .

3. Parentless child will transmit a packet to all terminals within its range stating that the link to its parent is broken. Packet Contains : SourceID = ParentlessChildID, Message Type $=13$

4. All mobile terminals(potential parents) that receive the packet in step 3(except children of parentless terminal) will reply to the message. Packet Contains : SourceID = PotentialParentID, DestinationID = ParentlessChildID, MessageType=14, Layer(of Potential parent)

5. Parentless terminal will acknowledge to one potential parent in the lowest layer based on its criterion. Packet Contains : SourceID = ParentlessChildID, DestinationID = NewParentID, MessageType $=15$

6. This new parent will forward this information to its parent towards the base station. The base station will forward it to the old parent so that it can update its records. Packet Contains : SourceID = NewParentID, DestinationID = BaseID, NewChildID = ParentlessChildID, MessageType=16 34 Revista da Sociedade Brasileira de Telecomunicac, . oes Volume 19, N'umero 3, Dezembro de 2004

7. The terminal that used to be parentless will also transmit this information to its children telling them to update their layer number. The children will transmit this information to their children and so on. Packet Contains : SourceID = NewParentID, Layer, MessageType $=17$ One good property about the oute update algorithm is that it will continue to provide us with routes that have a minimum number of hops. This is because the Route Determination Algorithm provides us with routes that have a minimum number of hops and if a mobile terminal changes positions, it will choose a new parent that has the smallest layer number.This means that the number of hops to the base station will still be minimal. Another possibility is that there is a change in the location of a mobile terminal which does not cause it to become out of range of its parent and its children. This may cause the routes to no longer have a minimum number of hops and for a greater amount of interference at the receiving terminals than is necessary. Therefore a mechanism needs to be designed so that routing paths can be updated periodically even when mobile terminals have not moved out of range of their parents or their children. The mechanism designed to accomplish this is to periodically rerun the route determination algorithm. 
This will be done every $\operatorname{Tr}$ seconds. $\operatorname{Tr}$ will be larger than $\mathrm{Tu}$ because the recalculation of all the routing paths using the route determination algorithm will create a large overhead in the system. By rerunning the route determination algorithm every $\mathrm{Tr}$ seconds we can ensure that the routing paths in the network remain in the manner designed over a long period of time.

\subsubsection{ROUTE UPDATE ALGORITHM EXAMPLE}

In this example we will be begin from the topology shown in Figure 7. Every so often, one of the terminals will move which may cause a link to break. This is illustrated in Figure 8. Therefore the Route Update Algorithm will be needed to _nd a new routing path for the terminal.

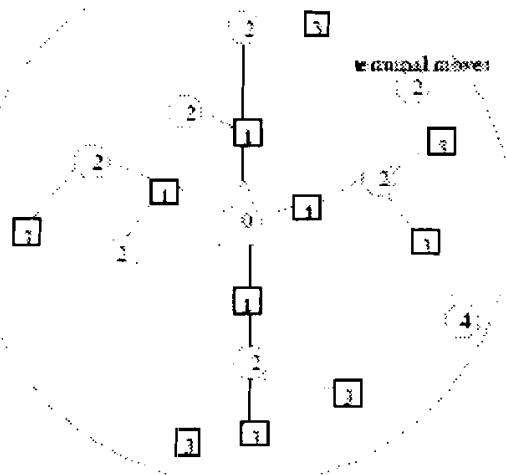

Figure 8. Movement of Mobile terminal triggers Route Update Algorithm

A short time later(= Tu 2 on average), when the terminal that moved checks to see if it is still within range of its parent, it will discover that it is not and will attempt to _nd a new routing path to the base station. Note that the child of the terminal who moved will also be out of range of its parent and it will also run the route update algorithm to _nd a new parent. Figure 9 shows the updated routing paths for the terminals upon completion of the route update algorithm. Also note that the layer numbers of the terminals are updated due to the new topology.

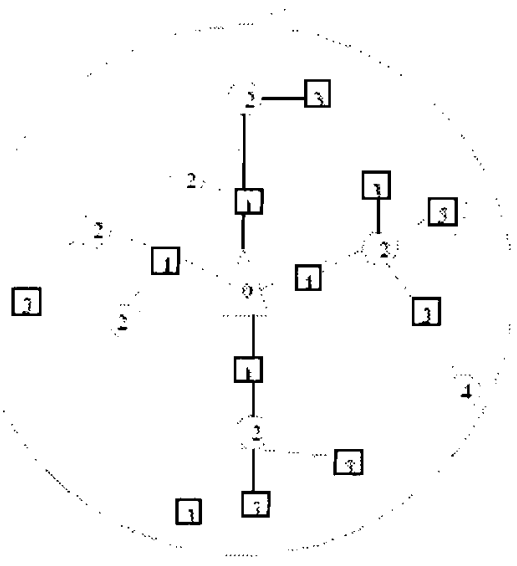

Figure 9. Convergence of Route Update Algorithm
A route determination algorithm has been designed to calculate routing paths for each mobile terminal to and from the base station, and a route update algorithm has been designed to update routing paths in case of a network topology change. Since routing paths have now been discovered, transmissions of packets to and from the base station can be made. Similarly, routing paths can be created for new terminals that are activated. Routing paths can also be deleted for terminals that are deactivated or that leave the network. For the sake of brevity, explicit descriptions of these algorithms will not be presented. Section 5 will discuss the numerical results obtained through simulation and analysis.

\section{SIMULATION RESULTS}

The radius of the cell $R$ used in the simulations is 100 . The number of mobile terminals $M$ in the cell is 200. Positions of mobile terminals will be randomly distributed within the cell. All simulations will be averaged upon 100 different realizations of positions of the mobile terminals and each realization will simulate 1000 timeslots. For the simulations, the noise power N0 will be set to 10,12 . The interference created by other transmissions will be treated as independent additive noise [?]. Average path loss for transmissions is inversely proportional to $\mathrm{d} 4$. Shadowing will be modelled using independent lognormal random variables with $\_=6 \mathrm{~dB}$. Packet arrivals will be Poisson distributed with an arrival rate of ${ }_{-}=0: 1$ packets per timeslot for each mobile terminal. The processing gain of the system $\mathrm{N}$ will be equal to 128 and we will be considering a chip rate equal to $1.2288 \mathrm{Mcps}$ as in a CDMA2000 $1 \mathrm{X}$ system. A spreading bandwidth of 1.25 $\mathrm{MHz}$ will be used which is equivalent to the bandwidth of one sub-carrier in a multicarrier CDMA2000 system. For all of the simulation results presented in the follow- ing sections, the routing paths are discovered using the route determination algorithm designed in this paper. All of the packets in the reverse link will be forwarded to the base station. The base station will be used to forward all packets to their eventual destination. The simulation of one cell will be used to model the overall network. Therefore, the effects of intercell interference will not be analyzed in this simulation. A power control mechanism will be used to ensure that the received SNIR is suf_ciently high so that the packets can be properly decoded but not too much higher so that the transmission is creating too much excess interference for other mobile terminals. In a multihop cellular network, the transmission range of each mobile terminal is decreased as compared to a traditional cellular network. The maximum transmission range for the multihop cellular network will be reduced to $I K$ of the maximum transmission range in a traditional single hop cellular network. For all of the simulations presented in the following sections, the results will be graphed with respect to the transmission range factor $\mathrm{K}$, so that the performance of the route determination algorithm can be compared for different maximum transmission ranges. Each simulation will be performed for the two variations of the route determination algorithm: choosing routes using the 
minimum distance criteria and choosing routes using the maximum distance criteria.

\subsection{SNIR}

The area surrounding the base station is the place where the lowest SNIR will occur within the cell. This is because every packet will eventually be forwarded to the base station. The maximal SNIR is achieved using a value of $\mathrm{K}=1$, which is equivalent to a single hop solution. This is because any other transmissions involving the forwarding of packets will just create excess interference at the base station. However, due to the design of minimum hop routing paths and the use of timeslots, only terminals that are at least two hops away from the base station will be transmitting at the same time as the children of the base station. This property of the routing algorithm minimizes the additional interference introduced by adding multiple hops. Figure 10 shows the signal to noise and interference ratioobtained when simulating the multihop cellular network with paths computed using the route determination algorithm. The _gure shows the SNIR at the base station which is the bottleneck of the system. From this _gure we can see that there is little change in the SNIR at the base station as we increase the transmission range factor(or decrease the transmission range of each terminal). We can also notice that choosing routes using the minimum distance criteria yields higher values of SNIR than when choosing routes using the maximum distance criteria. This is because using the maximum distance criteria involves choosing next hop choices that are further away(i.e. greater interference between terminal and next hop) which leads to larger use of transmission power and more interference. In terms of capacity the performance of the minimum distance criteria is better than the maximum distance criteria.

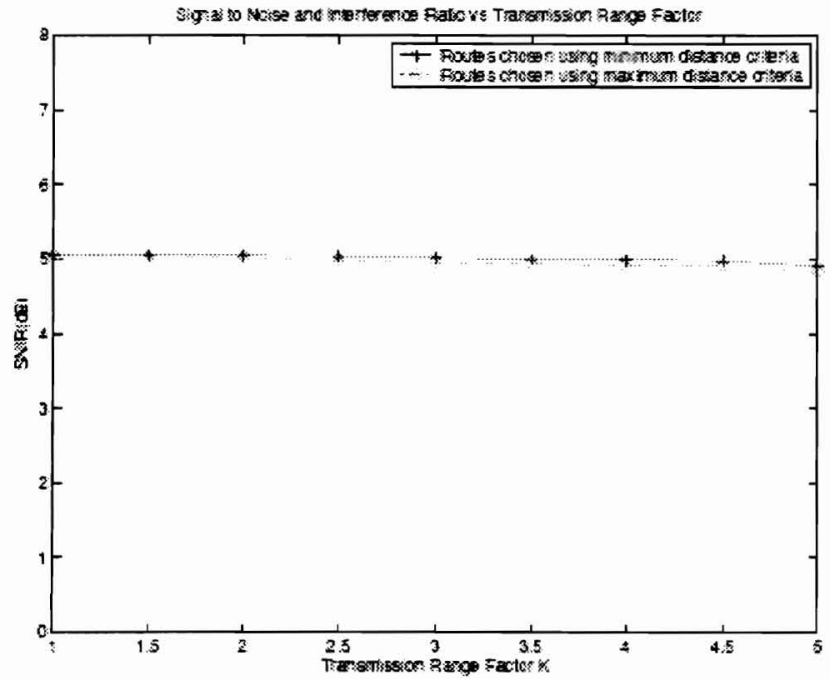

Figure 10. Signal to Noise and Interference Ratio vs Transmission Range Factor

From Figure 10, we can see that the SNIR decreases as the transmission range factor is increased. This is expected because as the maximum transmission range is decreased, mobile terminals in higher layers will be closer to the terminals in layer 1 . Their signals will therefore experience less path loss at the base station as they are closer to it and will create greater interference. The only terminals that create interference at the base station will be odd layered terminals because the even layered terminals will be receiving transmissions and will not be transmitting at this time. In fact the SNIR at the base station, is decreased by less than $0.3 \mathrm{~dB}$ from its optimum value even when decreasing the transmission range to one _fth its original value.

\subsection{DELAY}

Figure 11 shows a graph of the average hop count for packets of a mobile terminal to the base station versus the transmission range factor. It can be noted that the mean hop count for routes using the minimum distance criteria and maximum distance criteria is the same because both algorithms yield minimum hop routes. A small overall hop count is an essential property of the algorithm because it is important in many real-time applications that the end-toend delay for packets be small. From this gure, it can be observed that as the maximum transmission range decreases, the mean number of hops will increase as expected. When the transmission range factor is 1 , the number of hops to the base station is equal to one as expected because this is the single hop case. It can also be noted that even when the transmission range of a mobile terminal is decreased to one _fth of the range in a traditional cellular network that the mean number of hops will be equal to 5.43. Note that the average number of timeslots needed to reach the base station from a mobile terminal would be equal to ap-

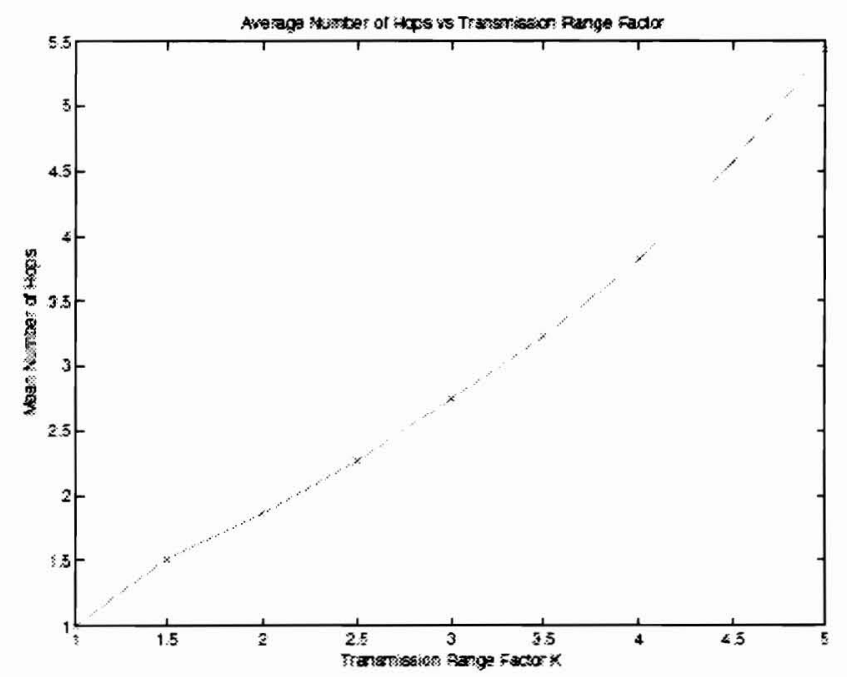

Figure 11. Mean Hop Count vs Transmission Range Factor

proximately 0.5 more than the mean number of hops. This is because a packet will have to wait an average of half a timeslot to begin being transmitted. Because packets from terminals in odd timeslots get transmitted to terminals in even timeslots and vice-versa, once a packet begins being transmitted, it will never have to wait another timeslot to be forwarded to the base station. In an ad hoc network, the end-to-end delay can be much larger because a base station is not present to forward packets to destinations that are far away. This is one advantage of the multihop cellular 
network over an ad hoc network. Figure 12 shows a graph of the maximum hop count from any mobile terminal to the base station for different values of the transmission range factor. For small $\mathrm{K}$ and suf_ciently large number of mobile terminals it can be shown that the maximum hop count for a minimum hop routing algorithm is equal to $2 \mathrm{~K} \square \square 1$. We can observe that for $\mathrm{K}<4$, that this is achieved. The reason that this is not achieved for $\mathrm{K}>4$ is that the transmission range is so small, that there are not enough terminals for this to be guaranteed. However, only in very odd network topologies will the maximum hop count be larger than $2 \mathrm{~K} \square \square 1$. Given the network topology the route determination algorithm always _nd routes with the minimum possible number of hops.

\subsection{POWER CONSUMPTION}

Figure 13 shows a graph of the average total power consumption expressed in decibels with respect to the transmission range factor. As the transmission range factor increases, the total power consumption within the network will decrease. Therefore, we can see that the total power consumption is much lower when using multiple hops than in a traditional single hop network. This _gure shows that when decreasing the transmission range by one _fth of the value in a single hop cellular, that a savings of $18 \mathrm{~dB}$ of power can be obtained throughout the network. This is an important result because mobile terminals rely on battery power andsuch a large savings will lead to a much longer battery life.

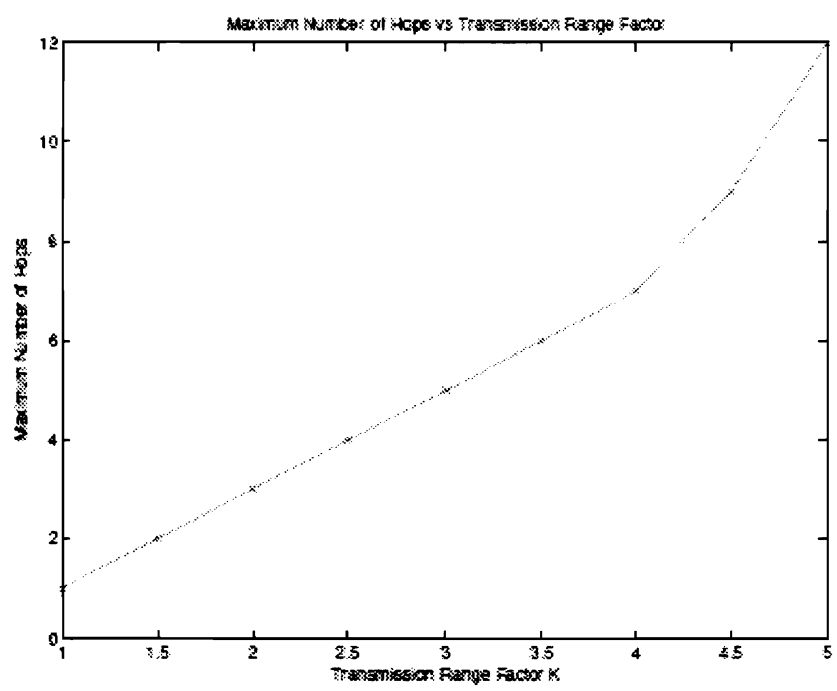

Figure 12. Maximum Hop Count vs Transmission Range Factor

This is due to the fact that the average power attenuation is proportional to $1 \mathrm{~d} 4$ and therefore any small decrease in the distance between the source and destination will yield a much smaller necessary transmission power. It can also be observed from this graph that the power consumed when using the minimum distance criteria is smaller than the power consumed when using the maximum distance criteria. This graph shows that the power consumption is always higher when using the maximum distance criteria. In fact, the power consumption when using the maximum distance criteria is greater than $28 \%$ than when using the minimum distance criteria for transmission ranges between 1.5 and 4 . Therefore, in terms of power consumption, the minimum distance criteria performs better than maximum distance criteria.



Figure 13. Average Total Power Consumption in $\mathrm{dB}$ vs Transmission Range Factor

\subsection{NETWORK LIFETIME}

Network lifetime will be de_ned as in [?] as the time until at least one mobile terminal loses all of its transmission power and is forced to deactivate. This will force the system to update routing paths using the route update algorithm and this creates overhead within the system. Therefore it is preferable to have a large network lifetime. Having a large network lifetime is a sign of fairness because it means that the power usage is distributed evenly among the different mobile terminals. The network lifetime for the multihop cellular network was measured and it is shown in Figure 14. In this simulation, each mobile terminal began with identical batteries with equal amounts of power. The network lifetime was de_ned as the number of timeslots needed for any mobile terminal in the cell to use all of its battery power. We can see that the network lifetime of the multihop cellular network increases as the transmission range factor increases. When performing the simulations, it was noticed that the mobile terminals that fail _rst are always the terminals within layer 1. This is as expected because mobile terminals in the _rst layer are in charge of forwarding the packets of all other mobile terminals. 


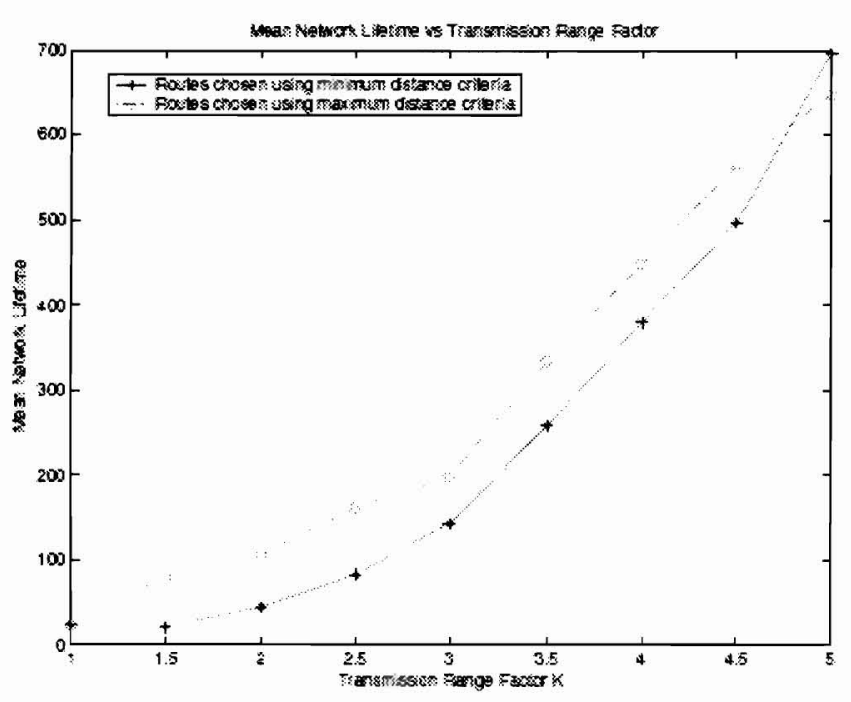

Figure 14. Mean Network Lifetime vs Transmission Range Factor

It can be be observed that the mean network lifetime when using the maximum distance criteria is higher than when using the minimum distance criteria. At rst glance this seems like a surprising result because the overall power consumption when using the maximum distance criteria is higher. However, the reason that the network lifetime is higher for the maximum distance criteria is that it is better at distributing the job of forwarding packets among many mobile terminals whereas the minimum distance criteria tends to allow some mobile terminals within a layer to do more forwarding of packets than others. Therefore, the maximum distance criteria creates routing paths that exhibit more fairness than the minimum distance criteria. This is especially true when the transmission range factor is small. Therefore, in terms of network lifetime, the maximum distance criteria performs better than the minimum distance criteria. At large transmission range factors, this effect is minimized and in fact the minimum distance criteria performs slightly better than the maximum distance criteria. In all of the simulations shown in this section with the exception of the network lifetime, the minimum distance criteria performed better than the maximum distance criteria. Also the performance of the system improved as the maximum transmission range of a terminal was decreased. In section 6 , conclusions will be formulated based on the results of these simulations.

\section{SUMMARY AND CONCLUSIONS}

In this paper, a minimum hop routing algorithm was designed and optimized for the multihop cellular network. Mechanisms to update routing paths in case of a network topology change were also developed. All transmissions in the multihop cellular network were made within timeslots and the base station was used to relay all packets to their destination. Simulations were performed on the multihop cellular network with routing paths calculated using two variations of the route determination algorithm. The route determination algorithm was judged based on various parameters including SNIR, delay, power consumption, and network lifetime. It was shown in this paper that when using minimum hop routing, a much lower power consumption can be obtained for the multihop cellular network than for a traditional single hop cellular network. It was also shown that the introduction of multiple hops causes only a very small reduction in SNIR. The reason that the decrease in SNIR was minimized is that minimum hop routes were chosen. This allowed interfering terminals within different layers to be as far apart from one another as possible, hence minimizing interference. We also illustrated that the end-to-end delay using the minimal hop route determination algorithm is much smaller than in a typical ad hoc network due to the introduction of a base station. The network lifetime of the multihop cellular network using the route determination algorithm to calculate routing paths was also found. A large network lifetime is important for the multihop cellular network because when a mobile terminal runs out of battery power and is forced to turn off, the routing paths will need to be recalculated and this creates additional overhead in the system. A large network lifetime also indicates fairness because power usage is being distributed more evenly between terminals in the network. We showed that the total power consumption within the network and network lifetime was greatly increased as the maximum transmission range of each terminal was decreased. It was also shown that the SNIR only exhibited a small decrease as the maximum transmission range of each terminal is decreased. Overall, the simulations illustrated that small maximum transmission ranges exhibited better performance than large transmission ranges. It was illustrated through most of the simulations that the minimum distance criteria for the route determination algorithm performed better than the maximum distance criteria in terms of capacity and power consumption. The only metric in which the maximum distance criteria performed better was in terms of network lifetime because it distributed the job of forwarding packets amongst more mobile terminals than when using the minimum distance criteria. Overall, the simulations illustrated that the minimum distance criteria performed better than the maximum distance criteria. While this paper led to the development of a minimum hop route determination algorithm that shows a substantial decrease in transmission power and only a minimal decrease in SNIR when introducing multiple hops, there are still some related issues that must be studied further. Firstly, a loadbalancing mechanism could potentially allow the forwarding of packets to be performed by a greater number of mobile terminals. This would lead to a system with greater fairness. Hence, the effects of a load balancing mechanism should be investigated for the multihop cellular network. Secondly, the introduction of path diversity using multipath routing could also increase the network lifetime and could minimize the number of times that the routing paths would need to be recalculated. This would lead to a reduction in overhead and a higher reliability of the system. Therefore, multipath routing should be considered for a multihop cellular network. Thirdly, the effects of the mobility of mobile terminals should be investigated because mobility cannot be neglected in the case of a high mobility environment. Fourthly, the reduction in the transmission ranges of mobile terminals will decrease the intercell 
interference in the multihop cellular network. Therefore, the reduction of intercell interference in a multihop cellular network as well as its effect on system capacity should be investigated. Finally, the performance of the multihop cellular network with the presence of asymmetric traf_c should be investigated. Because mobile terminals can now transmit on both frequency bands, it may be possible to transmit on the forward link using both the high and low frequency bands at certain times when the traf_c on the forward link is large and the traf_c on the reverse link is small. To accomplish this, an ef_cient scheduling mechanism for transmissions on the high and low frequency band for asymmetric traf_c would need to be designed for the multihop cellular network. This is a very interesting problem that should be investigated further.

\section{REFERENCES}

[1] K. Pepe and B. Vojcic, "Cellular multihop networks and the impact of routing on the snir and total power consumption," proceedings of Multiaccess, Mobility and Teletraffic for Wireless Communications 2002, June 2002.

[2] A. A. Kusuma and L. L. Andrew, "Minimum power routing for multihop cellular networks," IEEE Globecom 2002, 2002.

[3] Y. Lin and Y. Hsu, "Multihop cellular: A new architecture for wireless communications," IEEE Infocom. 2000.

[4] T. Cormen and C. Leiserson, "Introduction to algorithms," The MIT Press, pp. pp. 558-565, 1992.

[5] Z. Haas, "On the performance of a medium access control scheme for the reconfigurable wireless networks, "MILCOM" 97. pp. pp. 1558-1564, 1997.

[6] A. Viterbi and E. Zehavi, "Other-cell interference in cellular power controlled cdma," IEEE Transactions on Communications, volume 42, pp. pp. 1501-1504, 1994.

[7] D. Bertsekas and R. Gallagher, Data Networks, 2nd ed. Prentice-Hall, 1991, ch. 4.

[8] J. Chang and L. Tassiulas, "Energy conserving routing in wireless ad-hoc networks," Proceedings of IEEE Infocom 2000, 2000 .

Mark DeFaria is a Ph.D. candidate in the Department of Electrical and Computer Engineering at the University of Toronto. He received his B.A.Sc. in Engineering Science and his M.A.Sc. in Electrical Engineering at the University of Toronto, in 2001 and 2003, respectively. He was the recipient of the prestigious Edward S. Rogers scholarship for excellence at the graduate level. His research interests include QoS provisioning in mobile systems, ad hoc and sensor networks, and multihop cellular networks.

Elvino S. Sousa received the B.A.Sc. degree in engineering science and the M.A.Sc. degree in electrical engineering from the University of Toronto, Canada, in 1980 and 1982, respectively. He received the Ph.D. degree in electrical engineering from the University of Southern California, Los Angeles, in 1985. Since 1986, he has been with the Department of Electrical and Computer Engineering at the University of Toronto where he is now a Full Professor. Since 1983, he has performed research in spread spectrum systems. His current interests include the areas of high-speed CDMA systems, software radio, and ad hoc networks. He is Director of the wireless lab, University of Toronto, which has undertaken research in CDMA wireless systems for the past 15 years. He has been invited to give lectures and short courses on spread spectrum, CDMA, and wireless communications in a number of countries. He has spent sabbatical leaves at Qualcomm and Sony CSL, where he was the holder of the Sony Sabbatical Chair. Dr. Sousa was the Technical Program Chairman for PIMRC 95 and Vice-Technical Program Chair for Globecom99. 